\title{
De l'importance de la créativité entrepreneuriale. Le cas des petites entreprises
}

\section{The Importance of Entrepreneurial Creativity: The Case of Small Businesses}

\author{
Marc Jaillot ${ }^{1}$ \\ ${ }^{1}$ Université de la Polynésie française, marc.jaillot@gmail.com
}

RÉSUMÉ. La créativité est un domaine dont l'étude est plus récente que l'innovation et dont les contours et les contenus sont encore flous. La créativité et l'innovation sont souvent abordées conjointement pour décrire l'innovation orientée produit qui permet à une entreprise de détenir un produit «star », mais le sont beaucoup moins lorsqu'il s'agit d'étudier les combinaisons réussies de multitudes d'idées qui aboutissent à une réussite entrepreneuriale. Pourtant cette démarche créative peut être difficile à imiter et constituer de ce fait une protection de l'avantage concurrentiel. L'objectif de cet article consiste à questionner la notion de créativité propre à l'entrepreneuriat, afin de déterminer sa place et son importance au sein des petites entreprises. Une première partie consistera à préciser le concept de créativité entrepreneuriale et le contexte choisi. Nous pointerons ensuite les éléments mettant en avant l'importance de la créativité pour des entrepreneurs de PE, en nous appuyant sur une étude de cas empirique qui apportera une contribution à nos propos et ouvrira sur une discussion en dernière partie.

ABSTRACT. Creativity is a new field of research compared to innovation and the final boundaries of creativity have not yet been clearly defined. Creativity and innovation have often been jointly used to describe product innovation through which companies obtain a "star" product as the single best idea. However, they haven't been much used to study successful combinations of a multitude of ideas that result in entrepreneurial success. Nevertheless, this creative process can be hard to copy and can therefore be a competitive advantage. The purpose of this article is to explore the concept of entrepreneurial creativity to explain and describe its place and importance in small companies. We will first describe the concept of entrepreneurial creativity in a small business context. Then we'll examine features that highlight the importance of creativity for entrepreneurs of small companies. The third part will rely on a complementary qualitative empirical research to provide insights and discuss the boundaries of entrepreneurial creativity.

MOTS-CLÉS. créativité, créativité entrepreneuriale, management de l'innovation, entrepreneuriat, petites entreprises

KEYWORDS. creativity, entrepreneurial creativity, innovation management, entrepreneurship, small businesses

\section{Introduction}

La créativité est un domaine dont l'étude est plus récente que l'innovation et dont les contours et les contenus sont encore flous. La créativité est à la fois un processus et un résultat de ce processus qui permet de produire des idées. Quand elle ne concerne pas les produits et services destinés aux clients, elle est décrite comme la créativité organisationnelle. Woodman, Sawyer et Griffin (1993) considèrent la créativité organisationnelle comme un sous-ensemble de l'innovation. Pour Amabile (1988) la créativité est à l'origine de l'innovation à travers la production d'idées nouvelles et utiles et peut être considérée comme son antécédent. A travers son approche sur le leadership, Deschamps (2003) précise que stimuler, guider et soutenir l'innovation nécessite de mettre un accent tout particulier sur le développement des compétences, actuelles et futures, nécessaires à l'entreprise. Parmi ces compétences figurent la créativité, les savoir-faire, les valeurs, l'état d'esprit et les comportements générateurs d'innovations.L'innovation est souvent abordée sous son aspect résultat alors que la créativité semble plutôt représenter l'origine, la cause. Certains auteurs se focalisent d'ailleurs sur l'amont du processus, à travers ce qui favorise l'innovation, et s'intéressent à la capacité à innover (Parmentier, Szostak, 2015).

La créativité est alors présentée comme une capacité dynamique qui stimule l'activité innovante et conduit l'organisation à adopter des comportements risqués et des changements dans ses pratiques. Malgré cela, c'est le thème de l'innovation qui a fait l'objet d'un nombre important d'études académiques 
depuis les dernières décennies. De plus, la grande majorité des recherches a porté sur l'innovation technologique et plus récemment seulement sur l'innovation orientée organisation (Damapour, Aravind, 2012). Crossman et Apaydin (2010) montrent que la moitié des articles de recherche ne précisent pas le type d'innovation étudiée et, parmi celles qui le font, $3 \%$ seulement traitent de l'innovation organisationnelle. Dans une autre étude de littérature basée sur 7 revues et 342 articles parus entre 1992 et 2010, Keupp et al. (2011) ont identifié 25 articles seulement incluant l'innovation organisationnelle. Les études sur l'innovation semblent donc centrées sur l'innovation technologique, et les théories et pratiques se basent alors naturellement sur cette approche (Damanpour, Aravind, 2012) et nourrissent d'autant moins les questionnements sur l'aspect organisationnel. De la même manière, la créativité et l'innovation sont souvent abordées conjointement pour décrire l'innovation orientée produit qui permet à une entreprise de détenir un produit «star », mais le sont beaucoup moins lorsqu'il s'agit d'étudier les combinaisons réussies de multitudes d'idées qui aboutissent à une réussite entrepreneuriale, plus basée sur une innovation diffuse que sur the one best idea. Pourtant cette démarche créative, dont l'objet n'est pas forcément une innovation technologique, peut être difficile à imiter et constituer de ce fait une protection de l'avantage concurrentiel, alors même que la protection industrielle par brevet n'est pas mise en œuvre, comme c'est souvent le cas dans les petites entreprises (PE).

L'objectif de cet article consiste à questionner la notion de créativité entrepreneuriale en la confrontant à celle de l'innovation, afin de déterminer sa place et son importance au sein des PE. Une première partie consiste à préciser le concept de créativité entrepreneuriale et le contexte choisi. Nous pointerons ensuite les éléments mettant en avant l'importance de la créativité pour les entrepreneurs de PE, en nous appuyant sur une étude de cas empirique qui apportera une contribution à nos propos et ouvrira sur une discussion en dernière partie.

\section{Créativité, innovation et contexte entrepreneurial}

\subsection{La créativité entrepreneuriale}

La créativité que nous proposons d'étudier dans cet article est celle qui est propre à l'entrepreneuriat. L'entrepreneuriat est décrit comme un processus qui conduit à la création d'une nouvelle organisation (Gartner, 1995) et impulsé par un ou plusieurs individus s'étant associés pour l'occasion (Verstraete, 2003). Ces individus entreprennent souvent lorsqu'ils trouvent une opportunité d'affaires. L'entrepreneur(e)devient alors un individu qui perçoit une opportunité et qui crée une organisation pour la poursuivre (Bygrave, Hofer, 1991). Verstraete et Fayolle (2005) proposent une représentation de l'entrepreneuriat à travers quatre paradigmes : la création d'une organisation, l'opportunité d'affaires, l'innovation et la création de valeur. La notion d'entrepreneuriat fait souvent référence à la création d'entreprise. La littérature permet cependant de la définir dans un sens plus large, c'est-à-dire comme étant le phénomène au sein duquel évolue un (ou une) entrepreneur(e), que ce soit pour une création, une reprise ou un projet de développement propre à une organisation. Le processus intrapreneurial fait partie intégrante de l'entrepreneuriat (Carrier, 1997). L'auteure explique que les entrepreneurs et les intrapreneurs sont différenciés par la fait que l'intrapreneur actionne le processus entrepreneurial dans une organisation existante, à travers un nouveau projet par exemple, alors que l'entrepreneur est dans la phase de lancement ou de développement de l'entreprise nouvellement créée.

Le terme générique entrepreneur englobe les deux types. Leurs profils sont similaires et c'est surtout le contexte d'action qui les différencie. L'intérêt de s'intéresser à l'aspect entrepreneurial de la créativité réside dans le fait que cela amène à capter l'ensemble du phénomène créatif par le biais de l'entrepreneur qui est le détenteur des clés de développement de l'entreprise. L'entrepreneur, et en particulier l'entrepreneur de petite et moyenne entreprise (PME), ne sollicite pas uniquement sa créativité lorsqu'il crée un nouveau produit ou un nouveau procédé, mais aussi et peut-être surtout lorsqu'il organise son système de création de valeur. Comme exemples, Carrier (1997) montre qu'un entrepreneur doit souvent faire preuve de créativité pour trouver le capital nécessaire à son activité ou convaincre des investisseurs et des clients de l'intérêt de son produit ou service. Les compétences créatives des individus sont basées sur certaines 
caractéristiques comme la prise de risque, l'attitude face à l'incertitude ou à l'échec, l'autonomie qui sont aussi des attributs de l'entrepreneur (Bissola, Imperatori, 2011). La créativité est même considérée comme l'esprit de l'entrepreneuriat (Tsai, 2014) et joue un rôle important tout au long du processus entrepreneurial (Nyström, 1993).

\subsection{Le contexte entrepreneurial de la petite entreprise}

Le contexte entrepreneurial est important et le fait de s'intéresser à la petite entreprise pose au moins deux questions : qu'est-ce qu'une petite entreprise et pourquoi s'intéresser à ce type d'organisation ? Notre volonté de définir et d'étudier la petite entreprise présente à lui seul un intérêt car en choisissant le filtre qui formera le contour de la population étudiée on prédétermine le cadre de l'analyse et donc les résultats. La plupart des recherches donne l'impression que le choix n'incombe pas au chercheur, mais plutôt aux institutions qui ont déterminé les critères de segmentation de la population entreprise, d'abord au niveau national puis communautaire ou international. Ces critères sont plutôt quantitatifs car moins difficiles à mettre en place pour le recueil et l'analyse des données. Même si il y a une tendance forte à l'homogénéisation des critères et à la recherche de pertinence, comme par exemple à travers la catégorisation proposée par la Loi de modernisation de l'économie (Art. 51 LME, 2008-1354), on remarque que tous les pays du monde n'ont pas la même vision de ce qu'est une petite entreprise. D'autres typologies nous permettent d'ailleurs de segmenter différemment les entreprises (Julien, 1990). Toutefois, de nombreux auteurs montrent que la taille est certainement le facteur qui impacte et contingente le plus la structure d'une organisation (Mintzberg, 1982 ; Desreumaux, 1992), même si certains écrivent qu'il n'y a pas de «lois» concernant le lien entre la taille et les caractéristiques organisationnelles (Hall, 1972). La segmentation que nous avons choisie se base sur le critère taille, mais nous voulons cependant rester conscients de deux risques d'erreur possibles : celle qui consisterait à accepter à tort l'hypothèse de la spécificité de la petite entreprise et l'erreur de rejeter à tort des entreprises de type microentreprise ou entreprise de taille intermédiaire (ETI) sous prétexte qu'elles sont de taille différente (Torrès, 1997). Nous souhaitons aussi nous protéger d'un second biais qui consisterait à confondre les petites entreprises véritablement autonomes au sens du décret 2008-1354 avec celles qui sont juridiquement autonomes mais détenues par des firmes de plus grande taille. C'est le cas par exemple des unités légales, structures juridiquement indépendantes, qui peuvent cacher sous ce patronyme des formes peu autonomes d'entreprise car sous contrôle de grands groupes.

Nous finalisons le pourtour du segment d'entreprises ciblé grâce aux raisons qui nous poussent aujourd'hui à étudier la petite entreprise et qui résident principalement dans le rôle social donné aux entreprises et à l'entrepreneuriat : la capacité à générer des emplois et de la valeur ajoutée. Le développement de l'entrepreneuriat apparait de ce fait dans de nombreux programmes institutionnels, souvent comme élément contributeur possible au développement ou au redressement économique. Les objectifs recherchés se situent la plupart du temps au niveau de l'emploi et des richesses redistribuées, ce qui est plutôt justifié, de nombreuses recherches ayant en effet établi la corrélation entre entrepreneuriat et croissance (Howitt, 1998 ; Audretsch, Thurik, 2001). La Banque mondiale, l'OCDE, l'Union européenne, mais aussi les organisations non gouvernementales ou les réseaux d'entreprise encouragent la promotion de l'entrepreneuriat en tant qu'instrument fondamental de la croissance, du développement, du dépassement de la pauvreté et de l'insertion sociale. La commission européenne propose par exemple le plan Entrepreneuriat 2020 qui, comme composante de la stratégie Europe 2020, pose les bases « d'une croissance et d'une compétitivité intelligente, durable et inclusive » (Commission au parlement européen, 2013). Des mesures concrètes apparaissent dans certains domaines comme par exemple la politique éducative. Des travaux ont montré que la volonté de créer une entreprise peut être influencée en amont lors de la période d'apprentissage. La création du statut national d'Etudiant-Entrepreneur et d'un diplôme associé (Circulaire ministérielle EN-ESR, 2013) ou l'exposition à des jeux de simulation d'entreprise (Toutain, 2010) sont des exemples de réponses apportées pour motiver l'envie de créer une entreprise parmi les jeunes scolaires ou étudiants. 
S'intéresser à la petite entreprise c'est étudier 99,8\% des entreprises française de type PME (y compris microentreprises) qui représentent $46 \%$ des effectifs c'est à dire plus de six millions d'emplois équivalent temps plein (Insee, 2014). Si l'on s'intéresse seulement aux PME sans tenir compte des microentreprises, c'est moins de $4 \%$ des entreprises qui emploient près de $28 \%$ des salariés, soit 3,7 millions d'individus. Les entrepreneurs de microentreprises et PME sont donc détenteurs d'un levier puissant en termes d'emploi. Concernant les autres catégories, les 243 grandes entreprises (GE) et 5223 ETI emploient toutefois 4,5 millions de salariés, soit $30 \%$ du total. Le filtre de la loi de Pareto nous a donc conduits jusque-là à optimiser les actions vers peu d'entreprises qui représentaient le plus d'emplois. Mais, d'une part, ces entreprises de grande taille ont souvent les moyens humains et financiers pour se développer et innover et, d'autre part, il serait dommage de laisser de côté celles qui emploient plus de six millions de salariés. Ce sont de plus celles-ci qui ont le potentiel pour devenir de futurs grandes, même si, quelle que soit l'activité, la phase de lancement des premières années se fait avec une organisation réduite à quelques personnes la plupart du temps. Les créations d'entreprises débutent même sans salarié pour $95 \%$ d'entreelles, et parmi celles qui ont des salariés dès le démarrage, elles le font avec 2,7 personnes en moyenne (Insee-Sirene, 2014). Malgré cela, depuis près de dix ans, que ce soit en France ou au sein de l'Union européenne, les PME sont à la base de plus de $80 \%$ des nouveaux emplois créés, dont la moitié dans des entreprises de moins de 20 salariés. Les PME (y compris les microentreprises) emploient la majorité de leurs salariés dans les services destinés aux particuliers ainsi que dans le secteur des activités spécialisées scientifiques et techniques. Les microentreprises comptent $70 \%$ de leurs effectifs salariés dans les activités tertiaires. Globalement, les PME non microentreprises comptent en moyenne une trentaine de salariés. La majorité $(60 \%)$ a moins de 20 salariés et $12 \%$ seulement atteignent ou dépassent 50 salariés. Les activités exercées au sein des PME sont plus diversifiées que pour les autres catégories mais sont surreprésentées dans la construction et les industries de type traditionnelles. Dans le secteur de la construction qui compte pourtant une dizaine de grandes entreprises, $71 \%$ des salariés sont employés dans des PME (Insee, 2012).

\subsection{La réalité sur l'innovation dans les petites entreprises}

A la lecture des rapports on découvre que certaines caractéristiques sont propres aux petites entreprises et confirment que celles-ci peuvent être plus qu'un champ particulier d'observation, pour devenir «objet d'étude en soi, ontologiquement institué » (Marchesnay, 2003). Premièrement, la répartition de la création de valeur ajoutée (VA) montre que les petites et très petites entreprises créent moins de VA par salarié et par an que les ETI ou les GE (Insee, 2016). Les GE sont les plus grosses pourvoyeuses de VA avec $96,4 \mathrm{~K} € /$ salarié. Les ETI sont à 80,6 K€/salarié alors que les autres plus petites entreprises, PME et microentreprises génèrent 74,4 K€/salarié. Entre les PME et les microentreprises l'écart est aussi remarquable puisque ces dernières ont une plus forte capacité à créer de la VA 88,6 K€/salarié contre $65,0 \mathrm{~K} € /$ salarié pour les PME, pour un chiffre d'affaires par salarié similaire. Les PME sont donc les moins créatrices de VA/salarié. Ces chiffres sont des moyennes bien entendu, mais ces différences entre PME et microentreprises nous incitent à une analyse différenciée.

Nous constatons ensuite que les petites entreprises ne sont pas exportatrices. Plus de $56 \%$ de la valeur ajoutée est produite par environ 5200 entreprises (GE et ETI) qui réalisent $85 \%$ des exportations. Le secteur le plus concentré est celui des activités financières et assurances. Une trentaine de grandes entreprises y emploient 78\% des salariés. Insee Première (2014) met en avant que sont les entreprises exportatrices qui innovent le plus et les entreprises exportatrices ne sont pas des PME : seulement $15,5 \%$ de l'exportation en France est réalisé par les petites entreprises (PME y compris les microentreprises).

Le troisième point qui caractérise la petite entreprise est le fait que l'innovation ne semble pas être une problématique centrale, au sens d'une recherche de nouveauté à travers un processus élaboré. Que ce soit au niveau des dépenses de recherche, de la capacité à innover ou du dépôt de brevet, le constat est que cela est un domaine réservé aux GE et ETI. En 2014, 12567 demandes de brevets ont été déposées par des entreprises françaises à l'INPI pour une protection nationale, et sur ces 12567 demandes de brevets, 6715 (soit 53,4\%) ont été effectuées par 20 entreprises qui sont toutes des GE (INPI, 2015). Les dépenses 
de recherche en France sont de plus globalement très concentrées dans quelques branches de recherche. En 2013, les branches industrielles concentrent 79\% de la dépense intérieure de recherche et développement des entreprises (DIRDE). Parmi elles, l'industrie automobile, la construction aéronautique et spatiale ainsi que l'industrie pharmaceutique totalisent ensemble 34\% des dépenses. L'industrie automobile, avec $13 \%$ du montant de la DIRDE, est la première branche de recherche et on remarque que les industries ne représentent que $8,10 \%$ des entreprises (sociétés) et seulement $4,84 \%$ des entreprises créées (InseeSirene, 2014). Hors industrie et construction, huit entreprises sur dix réalisent des activités tertiaires et n'appartiennent donc pas à ces branches fortement impliquées dans l'innovation. Ces activités de services étaient jusqu'à maintenant peu étudiées alors qu'elles sont nombreuses et constituent des sources de création de valeur majeures dans nos économies hautement tertiarisées (Deslée, Guirod, 2012). Ce résultat peut cependant être nuancé selon le filtre choisi. Dire que les petites entreprises sont ou ne sont pas innovantes à partir de leur projet reste difficile en tant que tel et peut montrer des différences significatives selon que l'on prenne l'entreprise sous la forme d'unité légale ou au sens du décret de 2008. BPI France, dans son rapport sur les PME, préconise les critères liés aux projets de recherche et développement, ou le fait qu'elle publie au moins un brevet à l'INPI sur les deux dernières années d'exercice (BPIFrance-Lelab, 2015). Les études sur l'entreprise innovante restent complexes, partagées en premier entre l'approche sujet, basée sur le comportement et les activités d'innovation de l'entreprise, et l'approche objet qui est centrée sur le nombre et les caractéristiques des innovations produites (Manuel d'Oslo, 2005). Hors GE et ETI, les types d'entreprises qui innovent le plus sont soit les PME de plus de dix salariés et 20 ans d'existence, soit les jeunes nouvelles microentreprises de moins de cinq ans d'existence. Par rapport aux sources externes de l'innovation, substitut au développement interne, Archibugi, Cesaratto et Sirilli (1991) ont établi une relation entre la taille de l'entreprise et l'importance accordée à chaque type de source : l'acquisition de brevets et de savoir-faire joue un rôle plus significatif dans les grandes entreprises tandis que les biens intermédiaires acquis comme élément d'innovation sont le support privilégié des plus petites.

Enfin, point important en termes de spécificités, l'innovation est à mettre en corrélation avec le contexte. La grande entreprise, plus informée, définit son champ d'originalité par rapport à l'extérieur, aux marchés mondiaux alors que la PME est moins en mesure d'appréhender la nouveauté au sens absolu et définit l'innovation au sens relatif, par rapport à un contexte connu plus étroit, un marché plus localisé. L'innovation peut être une recombinaison d'idées anciennes aussi longtemps que l'idée est perçue comme nouvelle par les personnes impliquées, alors qu'elle pourrait apparaitre à d'autres comme une imitation de ce qui peut exister ailleurs, et cela prend en compte les idées, les individus, les relations et le contexte (Van de Ven, 1986). Les ressources pour innover peuvent alors facilement se situer au niveau de l'investissement (en équipement matériel par exemple) qui deviendra innovation pour l'entreprise par rapport à son niveau d'exploitation habituel (et aussi son marché).

On peut alors se poser la question de l'intérêt de mettre au cœur des débats l'innovation comme moyen de lutte concurrentiel alors qu'un pourcentage très réduit de petites entreprises semble en mesure d'effectuer une démarche complète et que les secteurs d'activité concernés par l'innovation technologique ne concerne qu'un nombre infime de sociétés qui disposent généralement des ressources et structures pour assurer le processus correctement. On constate que l'on sait communiquer, expliquer, former et accompagner les entreprises qui maitrisent déjà le processus, que l'on s'intéresse de plus en plus à celles qui sont en mesure de le faire et peuvent s'appuyer sur des structures d'aide à l'innovation. Mais que peut-on faire du point de vue de l'innovation avec les $99 \%$ des entreprises restantes ?

\section{Etude exploratoire empirique}

L'étude de la petite entreprise est donc intéressante pour comprendre son mode de développement qui ne passe pas forcément par l'innovation, ni par l'exportation, qui présente une capacité de création de valeur inférieure aux entreprises des autres catégories et dont les sources d'innovation diffèrent de par le contexte. Nous proposons d'approfondir la lecture de la petite entreprise à travers le filtre de la 
créativité qui peut expliquer une partie du mode de fonctionnement. Une étude exploratoire empirique met en évidence des aspects que nous ne trouvons pas dans la littérature. Notre objet de recherche consiste à expliquer et comprendre comment la créativité peut constituer un mode de fonctionnement particulier propre aux petites entreprises. Cela passe par l'identification des formes de créativité que l'entrepreneur génère et le recueil des informations sur la démarche créative. Une observation sur le terrain a consisté à questionner l'entrepreneur de petite entreprise sur la créativité en suivant l'activité principale pratiquée.

\subsection{Méthodologie}

Le choix d'une démarche exploratoire est justifié par le fait que nous ignorions ce que les entrevues allaient mettre à jour. Notre acquisition de connaissances s'est faite par des entretiens qualitatifs semidirectifs facilitant une discussion ouverte qui ne limite pas les propositions recueillies et permet un opportunisme méthodique (Girin, 1989). Les données obtenues ont pu être aussi bien quantitatives que qualitatives. Le questionnement a été réalisé sur le lieu de travail de l'entrepreneur afin de pouvoir, tant que faire se peut, conforter les informations extraites de l'entretien par une observation attentive de son environnement. David (2004) montre en effet la nécessité de décrire le contexte d'une étude de cas et de préciser de quel contexte il s'agit pour faciliter la validité et la fiabilité des résultats. Le choix est de limiter au maximum la participation de l'observateur. Seul le rôle de chercheur est clairement défini auprès des personnes interrogées. L'observation n'est cependant pas muette, et nous devons être conscient des parasitages dus à l'interaction entre le chercheur et l'observé.

En conformité avec notre choix d'étudier la petite entreprise, cette recherche terrain porte sur la petite entreprise telle que définie préalablement, c'est à dire un cœur de cible représenté par le segment des entreprises privées de type PME non microentreprise de moins de 50 salariés tous secteurs d'activités confondus. Un segment toutefois poreux ou nous nous autorisons à ne pas fixer de limites précises quant à la taille, comme vu auparavant. De la même manière, nous ne sélectionnons pas une activité particulière, en gardant à l'esprit que des résultats très différents pourraient être obtenus selon le métier de l'entreprise. L'observation passe par un recueil de données auprès du premier représentant de l'entreprise, l'entrepreneur, porteur de la dynamique entrepreneuriale. L'étude de la créativité est difficile dans le sens où elle nous ramène souvent à étudier la psychologie de l'entrepreneur alors que nous souhaiterions construire une explication des situations entrepreneuriales rencontrées. Notre approche ne consiste donc pas à nous focaliser sur l'acteur de l'entrepreneuriat et les traits qui le caractérisent, mais à avoir une vision des relations entre les individus et leur contexte en tant que situation entrepreneuriale (Schmitt, 2009). Nous abordons les problématiques des petites entreprises à travers les représentations des acteurs entrepreneuriaux dans un cadre de recherche basé sur une approche de type réel expérimenté (Schmitt, Filion, 2009). Le terrain choisi nécessite un contact avec le dirigeant, et la validation que l'entreprise et la personne ciblée sont conformes à notre population étudiée. Les principales difficultés sont d'obtenir l'accord d'une observation basée sur une relation franche et sincère, et de relater correctement ce que l'observation nous montre. La confidentialité peut être un frein à l'accès aux données en particulier sur les questions liées aux avantages concurrentiels ou aux données comptables. Pour cela, nous avons adopté une approche progressive de l'observation comme Lee (1993) le préconise. Un guide d'entretien semidirectif a été élaboré afin de pouvoir contrôler la trame de la discussion.

\subsection{Résultats}

La créativité est décrite dans la littérature comme un préalable de l'innovation au sein d'un processus commun à toutes les entreprises inscrites dans une démarche entrepreneuriale dynamique et efficace. Le terrain que nous avons étudié nous renvoie une image plutôt nuancée de la créativité générée au sein des petites entreprises. Nous avons choisi de nous focaliser uniquement sur un des cas étudiés qui explique assez bien notre propos et illustre en quoi la créativité est un aspect important de l'entrepreneuriat. Il s'agit d'une petite entreprise de 19 salariés qui a pour activité principale la projection de béton, le forage et le gunitage. Elle est assez représentative du point de vue de la taille puisque $60 \%$ des 
PME non microentreprises ont moins de 20 salariés avec une moyenne de 22 (Insee, 2012). Cette société du secteur de la construction existe depuis 1999 et réalise un chiffre d'affaires de 1,6 million d'euros. L'entrepreneur est arrivé dans cette activité grâce à des compétences acquises en tant qu'ancien artificier de l'armée française. L'observation a été peu guidée, l'entrepreneur abordant de lui-même les aspects qui nous intéressaient à travers son action et son discours. Le fait de se trouver immergé dans l'activité et de recueillir en même temps les informations données par l'entrepreneur a permis de corroborer efficacement le discours avec la situation. Trois situations de créativité assez spécifiques ont été identifiées sur le temps d'une séquence d'observation de quatre heures.

La première situation est un arbitrage économique assez classique de l'utilisation des facteurs de production travail et capital. Un choix de combinaison homme-machine est fait par l'entrepreneur en fonction de la disponibilité du moment de la force de travail. Certains salariés sont absents et d'autres n'ont pas toutes les qualifications, le planning fait apparaître un seul chantier principal qui nécessite d'optimiser l'utilisation de tous les ouvriers présents, l'entrepreneur dispose de plusieurs moyens matériels plus ou moins adaptés à la capacité nécessaire (pompe à bêton, tractopelle, compresseur...) et il effectue pour tout cela un choix d'allocation pour chaque chantier. Cette tâche peut sembler assez routinière et identifiable dans la plupart des entreprises. Il est difficile de la caractériser comme une innovation, même managériale, et pourtant le nombre de combinaisons possibles du système de production de cette petite entreprise peut très vite faire apparaitre des solutions jamais utilisées et considérées comme nouvelles pour l'entreprise.

Si on considère les trois variables suivantes : salariés (nombre, qualification, ancienneté), planning (date, heure, nombre de chantier différents à traiter sur une même période), matériels (type, capacité, disponibilité, transport) à combiner avec un contexte propre à chaque client, le nombre de combinaisons devient très vite très important et l'activité s'éloigne alors d'un processus routinier. Chaque chantier nécessite l'acquisition du maximum d'informations de la part de l'entrepreneur et entraine une organisation productive originale. On ne parle pas alors de nouveau processus puisqu'il s'agit en fait d'une multitude de possibilités qui conduit à mener à bien la mission. Il n'est alors pas possible singulièrement de parler d'innovation mais plutôt de mise en œuvre créative des éléments nécessaires à la réalisation du chantier. L'entrepreneur, continuellement fixé sur le management global de son activité, se concentre à ce moment-là sur la fonction production, à l'instar d'un responsable de chantier d'une grande entité. On peut alors parler de créativité d'activité, forme de créativité organisationnelle qui ne repose pas forcément sur un processus intentionnel d'innovation et qui entrerait dans le champ d'application théorique des méthodes de résolution de problème.

La deuxième situation observée dans cette entreprise montre l'utilisation d'un nouveau produit dans le processus de fabrication : un adjuvent liquide mélangé au produit fini béton. Celui-ci, bien que prévu pour d'autres types d'utilisations, permet une amélioration de la production à travers des critères classiques de baisse des coûts et de maintien d'une qualité conforme au cahier des charges. L'entrepreneur a récupéré fortuitement, mais en connaissance de ses problématiques, des informations sur un salon dédié à la construction et a ensuite généré l'idée d'utiliser un des produits découverts à cette occasion. Le bien a d'abord été testé par l'entrepreneur pour savoir s'il satisfaisait la demande de ses clients et apportait un avantage réel, notamment en termes de compétitivité. Le nouveau procédé, issu d'une démarche de type benchmarking, modifie le procédé de fabrication sans constituer une innovation réelle pour le marché. Il parait très proche d'une innovation produit, sans pour autant être protégée par un brevet. Par contre cette solution est nouvelle pour l'entreprise, sans certitude réelle toutefois quant à sa fiabilité. L'entrepreneur prend soin de demander de ne pas révéler cette méthode, qui doit rester secrète pour maintenir sa compétitivité. L'entrepreneur détient seul les clés de ce procédé. La créativité décrite ici ne correspond pas non plus aux processus d'innovation produit classique : pas de budget recherche et développement, pas de demande de brevet. Elle est propre à l'entrepreneuriat des petites entreprises où la culture du secret n'existe que par la forme centralisée de l'opérationnalisation de l'activité. 
La troisième situation est apparue lors du déroulement de l'activité. On peut la qualifier de problème urgent à résoudre en cours de réalisation d'un chantier. Le caractère d'urgence introduit la notion de temps car la chaîne complète de production était immobilisée entrainant les coûts fixes de non utilisation de l'actif immobilisé et des salariés. La décrire brièvement est important pour comprendre la situation : une surchauffe d'un des matériels (pompe à béton) réduit drastiquement la pression de pompage du béton en sortie qui ne permet plus la projection en hauteur sur les parois. L'entrepreneur est alors sollicité par ses salariés pour résoudre le problème. Une solution est trouvée en apportant un refroidissement complémentaire grâce à un dispositif très artisanal de jet d'eau sur le radiateur de refroidissement. Cette créativité actionnée apparait alors à l'observateur-chercheur plus comme un bricolage que comme une innovation, quand bien même nous pourrions la qualifier d'innovation frugale ou Jugaad. Le problème est pour autant résolu et permet peu de temps après le redémarrage de l'activité. Cette situation peut être perçue de la même manière que la situation numéro deux décrite auparavant, de type résolution de problème. Une observation attentive permet toutefois de faire ressortir un aspect tout autre. Si la créativité générée dans ce cas peut être considérée comme utile car le problème a été résolu, elle peut aussi être qualifiée d'absurde dans le sens où le matériel n'est pas utilisé conformément à ce qui est prévu par le constructeur. Les aérations obstruées par une bâche de protection contre les projections de béton, posée par les ouvriers, ont entrainé la surchauffe. L'entrepreneur l'a remarqué bien après le redémarrage de l'activité et en a fait la remarque aux salariés, oralement. Ceci nous amène au constat suivant, la créativité peut prendre une forme non attendue dans le sens où elle peut être inutile ou non pertinente dans les cas où une formalisation prévoyait un processus mais que celle-ci n'a pas été respectée. Au vu des nombreuses combinaisons possibles et à la difficulté pour un entrepreneur à appréhender tous les scenarii possibles, il est probable que ce type de situation se reproduise. Cette créativité peut être déclarée comme potentiellement superfétatoire car elle s'ajoute inutilement à l'activité.

Ces trois situation particulières nous font apparaitre la créativité sous des formes singulières qui ne sont pas forcément conformes à la description habituelle d'un processus de type créativité-innovation. A travers cette observation analytique on peut considérer que cette entreprise abrite une démarche effective de créativité entrepreneuriale alors qu'elle n'a ni projet dénommé recherche et développement, ni demande de brevet en cours. L'entrepreneur n'identifie d'ailleurs pas lui-même le lien entre sa créativité et la notion d'innovation, qu'il définit d'ailleurs uniquement comme une innovation produit.

\subsection{Discussion}

Il est donc difficile de marquer l'entreprise observée du sceau de l'innovation car elle ne répond pas aux critères d'une entreprise innovante. Pourtant on constate que l'entrepreneur apporte des éléments nouveaux de par sa créativité, et ceci de façon récurrente. Son activité est impactée par la démarche créative. La créativité actionnée correspond cependant à un cheminement entrepreneurial très éloigné de ce que l'on retrouve dans la littérature. Nous pouvons proposer de déconnecter la notion de créativité de celle d'innovation : l'entrepreneur de petite entreprise voit sa créativité positionnée au cœur de son métier sans pour autant que ce soit dans une intention systématique de mettre en œuvre un processus d'innovation. Cette créativité liée à l'activité peut compenser une routine mal établie, elle peut accompagner et faciliter le processus de production, elle peut aider à résoudre des problèmes, elle peut être inutile voir néfaste dans certains cas. Elle mériterait d'être consciemment évaluée par l'entrepreneur pour identifier son utilité réelle. La créativité peut conduire à l'innovation sous toutes ses formes, mais elle ne mène pas forcément aux résultats attendus des processus d'innovation classiques. La créativité entrepreneuriale n'est pas l'innovation. Par contre, elle peut constituer un préalable à la réflexion de chaque entrepreneur qui doit pouvoir évaluer, trier, sélectionner ce qui restera un élément de créativité sans suite ou pourrait devenir un axe stratégique pour l'entreprise et l'engager dans un des processus classique d'innovation. Sur cette frontière se situe peut-être, pour les entrepreneurs, un gisement de valeur qui mériterait d'être exploité (Figure 1). 


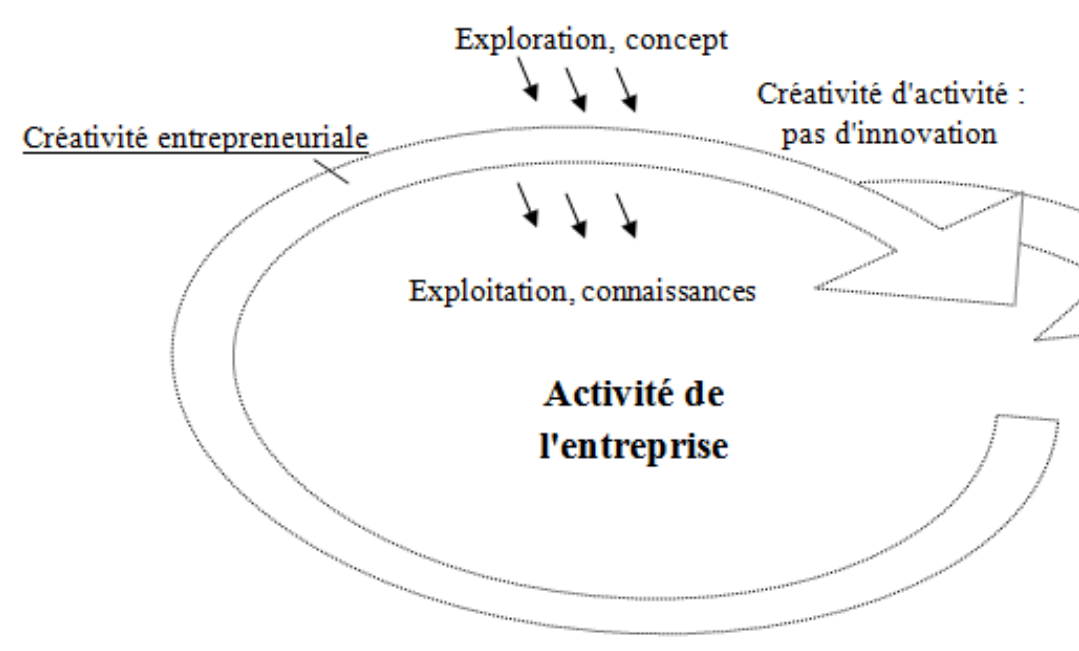

Figure 1. La créativité entrepreneuriale comme gisement de valeur de l'activité

La créativité entrepreneuriale montre au moins deux facettes : celle qui façonne l'activité, la modifie sans pour autant conduire à une démarche intentionnelle d'innovation, et celle qui est, ou deviendra, un préalable à une démarche d'innovation. La première, la créativité d'activité, peut provenir de la difficulté à mettre en œuvre l'activité, lorsqu'il est difficile, ou impossible, d'appliquer ou mettre en place des processus formalisés, ce qui conduit l'entrepreneur à générer des idées pour résoudre les problèmes. Cette difficulté à appliquer des routines établis trouve ses sources dans la variabilité des problèmes rencontrés, une insuffisance de formation et/ou de qualification des personnels, une gestion du temps et du caractère urgent des tâches à accomplir, un encadrement réduit au minimum et aussi une impression de rentabilité estimée accrue pour l'entrepreneur sous forme d'une performance personnelle perçue. Le fait de trouver des solutions ou de créer quelque chose de nouveau participe sans doute, comme l'a décrit Maslow (1943) à travers la hiérarchisation des besoins, à la réalisation de soi, à l'accomplissement de l'individu-entrepreneur.

Quelle que soit la forme de créativité générée, celle-ci constitue une approche créative propre aux entrepreneurs de petites entreprises car ils n'évaluent leur fonctionnement qu'à l'aune de leur propre conception d'une situation. Si l'entreprise se développe, la délégation et le partage de la décision peut modifier cela, et conduire à plus de formalisation et une approche créative différente, mais cela nous éloigne peu à peu du cadre de la petite entreprise. Le fait que l'activité soit réalisée avec peu de collaborateurs, pas forcément décisionnaires, oblige l'entrepreneur à introduire seul des processus organisationnels et à les gérer ensuite. La naissance d'une tension entre la créativité générée et les routines en place semble inéluctable et oblige à une ambidextrie plus ou moins appréhendée par l'entrepreneur.

Enfin la situation de créativité superfétatoire décrit dans la situation trois nous permet de réfléchir à la face cachée de la créativité. L'entrepreneur doit-il générer de la créativité pour refaire ce qui existe déjà ? Quel est l'intérêt d'inventer pour compenser simplement des routines mal exécutées ? La réponse est propre à chaque situation de chaque moment de l'activité de l'entrepreneur. Un calcul économique peut répondre à cette équation, prenant en compte d'un côté ce que coûte et rapporte la mise en place et l'application d'un processus ou d'une routine, et de l'autre l'économie de cette non mise en œuvre et le coût de la réponse créative. Le calcul nécessite toutefois de pouvoir anticiper l'émergence d'une réponse créative, ce qui est difficile à garantir surtout si l'entrepreneur n'a pas mis cette réflexion au cœur de sa démarche entrepreneuriale. Pour ce calcul économique on a donc d'un côté un coût déterminable et certain, si l'entrepreneur met en place un processus et tout ce qu'il faut pour l'appliquer, et, de l'autre, un coût difficilement chiffrable d'une solution créative aléatoire par définition. Si le calcul des coûts est difficile, la mesure des gains l'est tout autant. Pour effectuer cette mesure, l'entrepreneur peut arbitrer en introduisant dans sa démarche la notion de risque à travers une matrice d'évaluation (chiffrée ou non) des risques par exemple. Face à une situation complexe, l'entrepreneur adopte alors une attitude de choix. "Il ne choisit pas forcément de faire ce qui lui plait, mais choisit ce qui lui plait de faire ». Il peut 
dès lors aborder sa démarche créative avec les outils d'aide à la gestion du risque ou de l'incertitude (Merad, 2010). L'objectif est de fermer son univers décisionnel en tenant compte de tous les aspects pouvant influencer sa décision que ce soit au niveau stratégique, tactique ou opérationnel (Ansoff, 1965).

L'évolution des systèmes d'information facilite l'accès aux informations pour l'entrepreneur qui voit ses difficultés réduites pour opérer ses choix. L'externalisation de la mémoire par exemple rend l'information mieux disponible dans toute situation entrepreneuriale particulière qui apparait, au moment et à l'endroit où elle apparait. Un mode de fonctionnement différent risque d'apparaitre entre ceux qui savent exploiter les nouvelles technologies de l'information et de la communication et ceux qui, pour des raisons diverses, s'en sont trouvés à mis à l'écart. En poursuivant encore notre réflexion, nous pouvons toutefois nous questionner sur le respect des processus formalisés en place qui rendent très efficace le système de production, mais qui peuvent aussi devenir contraignants pour la démarche créative de l'entrepreneur. En effet, même si celle-ci parait peu pertinente lorsqu'elle vient compenser le non-respect d'un processus déjà établi, une créativité superfétatoire peut aussi amener l'entrepreneur à faire émerger des formes de sérendipité.

\section{Conclusion}

Cet article a pour objectif d'ouvrir un peu plus la boite noire de la créativité et apporter des éléments explicatifs quant à sa place au sein des petites entreprises. La confrontation des données macro sur la petite entreprise avec celles plus détaillées de l'observation terrain nous offre une étude grand-angle avec un zoom sur la créativité entrepreneuriale. Tenir compte de la créativité pour expliquer l'entrepreneuriat semble très pertinent à la vue de ces trois situations et nous permet de proposer le désassemblage des concepts de créativité et d'innovation, la créativité ne menant pas forcément à une innovation. Les formes de créativité qui peuvent apparaitre nous incitent à réfléchir sur la problématique de l'ambidextrie dans les petites entreprises. Si on utilise le filtre proposé par March (1991), il est aussi important pour un entrepreneur d'être capable d'explorer et de recueillir des nouvelles connaissances que de les exploiter. L'exploration et l'exploitation sont essentielles pour assoir la compétitivité présente et future et nécessitent une ambidextrie pour équilibrer les tensions qui en résultent (Ingram et al., 2008). L'entrepreneur de petite entreprise orchestrant les deux, il lui faut à nouveau faire preuve de beaucoup de créativité pour mener tout cela de front. Au-delà du débat sur l'importance de la créativité dans la PE, nous montrons aussi la difficulté méthodologique qui apparait lorsque l'on souhaite recueillir et exploiter des éléments probants sur le sujet de la créativité, les situations entrepreneuriales étant diverses et variées et la compréhension du terrain pas toujours évidente. Enfin, pour cet article, nous avons utilisé un seul cas d'entreprise pour compléter notre approche théorique et répondre à notre question. Il sera intéressant de poursuivre cette étude avec une quantité plus importante d'entreprises afin d'une part de construire une représentation plus théorique des formes de créativité existantes au sein des petites entreprises et aussi de comparer les résultats en fonction de facteurs discriminants identifiés.

\section{Références}

AMABILE T.M., A Model of Creativity and Innovation in Organizations, Research in Organizational Behavior, vol. 10, $\mathrm{n}^{\circ}$ 1, p. 123-167, 1988.

ANSOFF H.I., Corporate Strategy: Business Policy for Growth and Expansion, McGraw-Hill Book, 1965.

Archibugi D., Cesaratto S., Sirilli G., Sources of Innovative Activities and Industrial Organization in Italy, Research Policy, vol. 20, n 4, p. 299-313, 1991.

AUDRETSCH D.B., THURIK R., Linking Entrepreneurship to Growth (Lier entrepreneuriat et croissance), OECD Science, Technology and Industry Working Papers, 2001/2, OECD Publishing, 2001.

BISSOLA R., IMPERATORI B., Organizing Individual and Collective Creativity: Flying in the Face of Creativity Clichés, Creativity and Innovation Management, vol. 20, $\mathrm{n}^{\circ}$ 2, p. 77-89, 2011. 
BYgraVe W.D., Hofer C.W., Theorizing about Entrepreneurship, Entrepreneurship Theory and Practice, vol. 16, $\mathrm{n}^{\circ} 2$, p. 13-22, 1991.

CARRIER C., De la créativité à l'intrapreneuriat, Presse de l'université du Quebec, Sainte-Foy (Quebec), 1997.

Crossman M.M., Apaydin M., A Multi-Dimensional Framework of Organizational Innovation: A Systematic Review of the Literature, Journal of Management Studies, vol. 47, n 6, p. 1154-1191, 2010.

Damanpour F., ARAVind D., Managerial Innovation: Conceptions, Processes, and Antecedents, Management and Organization Review, vol. 8, n² 2, p. 423-454, 2012.

DAVID A., ThIÉTART R.A., Méthodes de recherche en management, $4{ }^{\text {ème }}$ édition, Dunod, Paris, 2004.

DESCHAMPS J.P., Innovation and Leadership, The International Handbook on Innovation, Elsevier Science, p. 815-834, 2003.

DeSREUMAUX A., Structures d'entreprise : analyse et gestion, Vuibert, Paris, 1992.

DesleE C., Guirod P., La combinaison de connaissances pour innover. Le cas de la création de services d'assurances innovants pour le distributeur, Revue française de gestion, vol. 2, p. 95-110, 2012.

GARTNER W.B., Aspects of organizational emergence, Bull J., ThOMAs H., Willard G., Entrepreneurship: Perspectives on Theory Building, Pergamon, London, vol. 70, 1995.

GIRIN J., L'opportunisme méthodique. La recherche action en action, Journée d'étude AFCET, Ecole centrale de Paris, 1989.

Hall H., Organizations: Structure and Process, Englewood Cliffs, N.J., Prentice-HaLl., TorRes O., 1997, Pour une approche contingente de la spécificité de la PME, Revue internationale PME : Économie et gestion de la petite et moyenne entreprise, 10(2), 9-43, 1972.

HowITT P., Endogenous Growth Theory, MIT Press, Cambridge, 1998.

Ingram A.E., Lewis M.W., Andriopoulos C., Gotsi M., Innovation Tensions and Organizational Ambidexterity: Toward a Collective Paradox Frame, Academy of Management Proceedings, vol. 1, p. 1-6, 2008.

JULIEN P.-A., Revue internationale PME : économie et gestion de la petite et moyenne entreprise, vol. 3, n 3-4, p. 411-425, 1990.

Keupp M.M., Palmié M., Gassmann O., The Strategic Management of Innovation: A Systematic Review and Paths for Future Research, International Journal of Management Reviews, vol. 14, n 4, p. 367-390, 2011.

LEE R.M., Doing Research on Sensitive Topics, Sage, Thousand Oaks, CA, 1993.

MARCH J.G., Exploration and Exploitation in Organizational Learning, Organization Science, vol. 2, n 1, p. 71-87, 1991.

MARCHESNAY M., La petite entreprise : sortir de l'ignorance, Revue française de gestion. vol. 144, n 3, p. 107-118, 2003.

Maslow A.H., A Theory of Human Motivation, Psychological Review, vol. 50, n 4, p. 370-96, 1943.

MERAD M., Aide à la décision et expertise en gestion des risques, Lavoisier, Paris, 2010.

MinTZBERG H., Structures et dynamique des organisations, Edition des organisations, Paris, 1982.

NYSTRÖM N., Creativity and Entrepreneurship, Creativity and Innovation Management, vol. 2, n 4, p. 237-242, 1993.

PARMentier G., SzOSTAK B., La créativité organisationnelle vue comme sous l'angle du management stratégique: proposition du concept de capacité créative d'une organisation, Communication AIMS, XXIV Conférence Internationale de Management Stratégique, Paris, 2015.

SCHMiTT C., Les situations entrepreneuriales: proposition d'une nouvelle grille d'analyse pour aborder le phénomène entrepreneurial, Revue Economique et Sociale, p. 11-25, 2009.

SCHMitT C., FILION L.-J., Le réel expérimenté pour explorer la TPE: apports conceptuels et méthodologiques, Management \& Avenir, vol. 30, p. 207-226, 2009.

TORRES O., Pour une approche contingente de la spécificité de la PME, Revue internationale PME : économie et gestion de la petite et moyenne entreprise, vol. 10, n 2, p. 16, 1997.

TOUTAIN O., Apprentissage expérientiel et métacognition dans l'éducation à l'entrepreneuriat, Thèse de doctorat, Université de Lyon, 2010. 
TSAI K.C., Creativity is the Spirit of Entrepreneurship, International Journal of Business and Social Sciences, vol. 2, $\mathrm{n}^{\circ} 1$, p. 106-115, 2014.

VeN A.H., Central Problems in the Management of Innovation, Management Science, vol. 32, n 5, p. 590-607, 1986.

Verstraete T., Fayolle A., Revue de l'Entrepreneuriat, vol. 4, nº 1, 2005.

Verstraete T., Proposition d'un cadre théorique pour la recherche en entrepreneuriat, Editions de l'Adreg, Paris, 2003.

WoOdman R.W., SAWYER J.E., GRIFFIn R.W., Toward a Theory of Organizational Creativity, Academy of Management Review, vol. 18, n², p. 293-321, 1993.

\section{Autres sources}

BPI France-Le lab, PME 2015, Rapport annuel sur l'évolution des PME. Observatoire des PME. http://library.bpifrancelelab.fr/PME15, 2015.

Circulaire ministérielle EN-ESR, Circulaire sur la création du statut national d'Etudiant-Entrepreneur. 18 mars 2013.

Insee, Insee-Sirene-Ree, Stock d'entreprise et entreprises créées en 2014, France et Insee Première n 1521, octobre 2014. 